\title{
Automated Non-Invasive Identification and Localization of Focal Epileptic Activity by Exploiting Information Derived from Surface EEG Recordings
}

\author{
Amir Geva1 , Merav Ben-Asher'1, Dan Kerem², \\ Mayer Aladjem ${ }^{1}$ and Alon Friedman ${ }^{3}$ \\ ${ }^{1}$ Dept. Electrical Engineering, Ben Gurion University in the Negev, Be'er Sheva \\ ${ }^{2}$ School for Marine Sciences, University of Haifa, Haifa \\ ${ }^{3}$ Departments of Physiology and Neurobiology, Faculty of Health Sciences, \\ Ben Gurion University in the Negev, Be'er Sheva,
}

Israel

\section{Introduction}

Non-invasive epileptic source localization techniques, relying on interictal activity in the EEG and mainly motivated by the need for presurgical localization of the epileptic focus in intractable focal epilepsy, have been widely studied and validated (Blume et al., 2001; Ebersole, 2000; Gavaret et al., 2009; Lantz et al., 2003; Michel et al., 2004; Zumsteg et al., $2005,2006)$. Source localization methods most often utilize electric source imaging (ESI), a technique which applies various mathematical algorithms and constraining neurophysiological hypotheses on scalp electroencephalographic (EEG) recordings in order to reconstruct the three dimensional brain electrical activity (reviewed in Michel et al., 2004). Correlation of such modeling with actual intra-cerebral recordings is not always straightforward and some focal deep sources do not seem to have surface representation sufficient for stable modeling (Merlet \& Gotman, 2001). Advantage may also be taken of extracranial magnetic fields (MEG) which like scalp electric potentials are due to current density distribution that arises from post-synaptic processes of underlying neuronal populations. The MEG forms the basis for the various versions of low resolution brain electromagnetic tomography (LORETA), which have been advanced to tackle the solution of the inverse problem (the $3 \mathrm{D}$ reconstruction of electric neuronal activity based on extracranial measurements) (Pascual-Marqui, 2002).

Metabolic imaging investigations are considered not suitable to study the temporal dynamics of seizures due to poor temporal resolution. Yet, a new and rapidly developing non-invasive imaging technique of EEG-correlated functional magnetic resonance imaging (EEG-fMRI) which maps regional changes in cerebral oxygenation and blood flow that are time-locked to interictal epileptiform discharges (IED) identified on the simultaneously recorded EEG, can identify metabolic activations correlated to IEDs (Vulliemoz et al., 2009). 
Due to its high temporal resolution, EEG is the most frequently used non-invasive method for the diagnosis of epilepsy and for localizing its source. However, the cortical potentials are severely attenuated by bone and soft tissues as they spread to the scalp, requiring amplification that facilitates contamination by muscular and external potentials with a low signal-to-noise ratio. The scalp recording is hence a mixture of neural signals, artifacts and noise, causing the identification of epileptic activity and its origin to be a challenging quest, even for experienced clinicians. Thus, there is still a great need for efficient signal processing methods in order to clean the signal and localize its neural sources.

During the last decade, blind source separation (BSS) has been successfully used for various EEG-processing applications, including artifact rejection (Bouzida et al., 2005; Frank \& Frishko, 2007; James \& Gibson, 2003; Shoker et al., 2005; Xue et al., 2006), discrimination of mental tasks in healthy individuals (Chiappa \& Barber, 2005; Liu et al., 2004; Makeig et al., 2004; Sutherlanda \& Tanga, 2006), localization of abnormal brain signals (Latif \& Sanei, 2005; Richards, 2004; Zhukov et al., 2000) and diagnosis of Alzheimer's disease (Cichocki et al., 2005).

Several attempts have been made to extract epileptic signals using BSS. It has been demonstrated that removal of artifacts using BSS improves seizure detection (Liu et al., 2004). While many studies addressed the problem of seizure recognition, identifying the epileptic source out of separated components was in most cases done subjectively by visual inspection (Hesse \& James, 2005; James \& Lowe, 2000; Kobayashi et al., 1999). Other workers, e.g. Jing \& Sanei (2007), employed clinical information concerning the location of the epileptic focus and prior assumptions regarding its frequency. Faul and colleagues (2005) and Jarchi and colleagues (2009) developed a method for seizure source localization based on a complexity measure and singular value fraction. These studies relied on the different temporal characteristics of normal EEG activity and pre-ictal/ictal signals, the latter consisting of slower, rhythmic activity, spikes or spike and wave complexes. The flexibility of fuzzy clustering algorithms (Bezdek, 1981) enables them to uncover hidden states without forcing a priori constraints on cluster features. This has been used by researchers to identify brain states based on EEG activity, following previous feature extraction. Geva and Kerem (1998) used a fuzzy clustering algorithm (Gath \& Geva, 1989) following wavelets analysis on pre-ictal recordings to classify brain states and forecast epileptic seizures. Chang and Po (2005) used wavelet analysis followed by fuzzy k-means clustering to track brain states of meditation from EEG recordings. Others had since used a similar clustering procedure for classifying polymorphic seizures according to their similarity in feature space (Meier et al., 2008).

With the rising popularity of BSS for EEG analysis, its ability to extract features for further clustering was highlighted. In fact, BSS is able to distinguish between overlapping clusters, a feat not possible otherwise. Hallez and colleagues (2007) have shown that the use of BSS to filter noise and artifacts prior to dipole estimation and clustering of the dipoles improved the localization. Makeig et al (2004) clustered scalp maps and activity power spectra of independent components drawn from EEGs of subjects performing visual tasks. They were able to identify clusters with functionally distinct activity patterns such as eye movements and muscle artifacts.

Since the epileptic activity patterns are likely to change over time, attempting to identify them by their temporal/spectral structures alone may prove problematic. Imposing constraints on the nature of the epileptic activity may also reduce the likelihood of identifying it. Thus, there is a call for an unsupervised method that will blindly extract and 
localize the epileptic signals. Since in patients with partial/focal epilepsy there is a single (or a few) focus (i) which triggers an epileptic seizure, it was decided to search for sources with a similar spatial distribution on the scalp, but not necessarily with similar temporal characteristics. For this purpose, a method is here proposed which combines BSS and cluster analysis procedures. The BSS procedure is used to separate the temporal components of the epileptic source from noise and background neural activity. Then a cluster analysis procedure is implemented on topographic maps of the BSS components. The motivation for the latter is the fact that focal epileptic activity tends to originate from the same small brain region (or neuronal population), which is expected to produce a cluster of spatially restricted (not scattered) maps of the epileptic BSS components on the scalp. Even if and when the pre-ictal activity is projected from the source to other distinct and restricted brain locations such as the contra-lateral lobe (Baumgartner et al., 1995), it is still expected that member maps of the 'epileptic-source cluster' would be the least expanded. The method is tested on twelve patients with source-localized focal epilepsy who were video-monitored as part of their pre-surgical evaluation. The obtained results show that the method is a promising tool for accurate detection of focal activity on the scalp.

In Section 2, the method is derived and in Section 3 results of its application on the EEG of epileptic patients are validated against the clinical source localization. Conclusions are provided in Section 4 .

\section{A method for scalp localization of the epileptic zone}

In Section 2.1, the data acquisition and preprocessing steps are described then in Section 2.2, topological maps of the BSS components are constructed for EEG time windows, which are likely to contain information on the epileptic focus. In Section 2.3, a clustering procedure of the maps is developed and finally, by employing general clinical knowledge on an epileptic focus, a procedure is derived for the identification and localization of the latter in a given patient.

\subsection{Data acquisition and EEG preprocessing}

Data acquisition and noise reduction preprocessing of the EEG signals have crucial influence on the efficiency of the BSS and cluster analysis procedures. From each of 12 patients several (2-7) EEG records of variable lengths (0.8-35.5 min) were obtained, up to but excluding the seizure (see Table 1). EEG was recorded from 27 electrodes placed according to the 10-20 system, using a 128-channel digital acquisition unit (Bio-Logic, USA). The sampling rate was set to $256 \mathrm{~Hz}$ and an input bandpass filter with the range of $0.3-100 \mathrm{~Hz}$ was applied. All electrodes were referenced to a common electrode (FCz).

EEG recordings from the scalp are severely contaminated with noise and artifacts, since the high resistance of the skull attenuates the neural signals, resulting in low signal-to-noise ratios. Moreover, there are many potential localized non-neural sources of electrical activity on the scalp, such as eye, eyelid, tongue or jaw movements, scalp muscle activity and cardiac activity, that produce scalp potentials as large as, or even larger than the EEG in general and the epileptic activity in particular (Nunez \& Srinivasan, 2006). At very low frequencies, muscle movement artifacts mainly overpower the neurological signals. Since epileptic activity is known to occur at relatively high frequencies, a 1.5-40 Hz finite impulse response bandpass filter (Proakis \& Manolakis, 2007) was also applied on the EEG signals. 
Brain activity is constantly changing, resulting in changes of the statistical properties of the EEG signal (Sanei \& Chambers, 2007). In order to attain signal stationarity which is required by the BSS methods and in order to minimize loss of information, the EEG processing was replicated on 50\% overlapping windows (Delorme \& Makeig, 2004) of 60 seconds duration, a length found suitable in a preliminary study.

Fig. 1 presents pre-processed EEG during the final five seconds of a $60 \mathrm{~s}$ window of Patient \#2 (Tables 1 and 2). Pre-seizure epileptic activity is observed in temporo-occipital electrodes bilaterally. The suspected epileptic focus in this patient was the left temporal lobe (predominantly recorded in electrode T5). This was also validated using 64 electrode subdural grids and sub temporal strip (8x2) electrodes, implanted prior to resection of the temporal lobe.

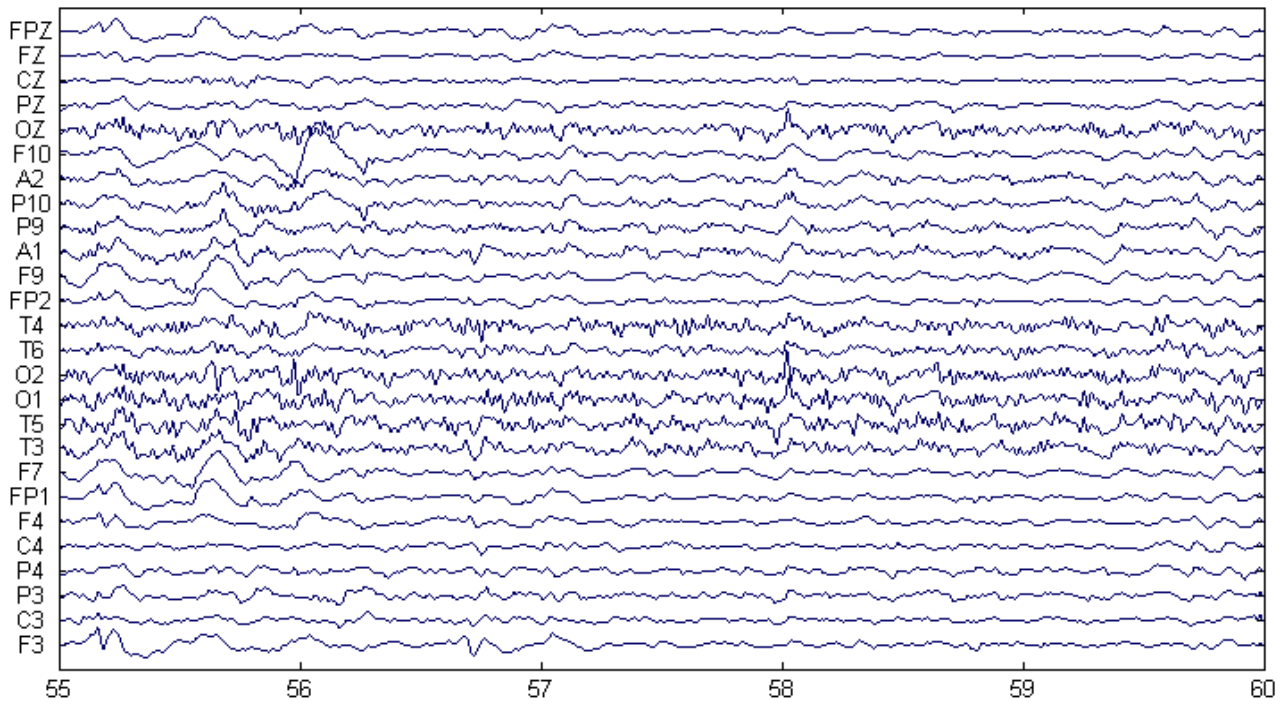

Fig. 1. Preprocessed EEG signals for patient \# 2.

\subsection{Topological maps of BSS components}

In the first stage of the presented method, BSS was employed for the reconstruction of neural (true EEG) and extra-neural components in the filtered electrical recordings. The input for the BSS procedure was the pooled EEG recordings (all pre-seizure records) of each patient. As mentioned above, pre-ictal and inter-ictal scalp recordings are a mixture of normal neural activity, epileptic neural activity, non-neural potentials (e.g. scalp and eye muscles) and external artifacts. Using the linear BSS method, it was assumed that the measured activity is a weighted sum of multiple independent sources. Delorme \& Makeig (2004) have shown that the linear model is a good approximation of the source mixing into the EEG recordings. In addition, it was concluded that the electrical fields of the recorded EEG are practically independent because neural and extra-neural activities are anatomically and physiologically separate processes (Hyvärinen \& Oja, 2000), which motivated the use of independent component techniques (Hyvärinen et al., 2001) for BSS in this work. 
For the present case, the linear BSS model can be expressed as follows in matrix notations:

$$
\hat{\boldsymbol{s}}(t)=W x(t),
$$

where vector $x(t)$ comprises the recorded EEG signals $\left\{x_{j}(t)\right\}_{j=1}^{n}$ from each of $n$ electrodes, $(n=27$ in this application) of a given $60 \mathrm{sec}$ time window of a given patient, vector $\hat{s}(t)$ with elements $\left\{\hat{s}_{i}(t)\right\}_{i=1}^{n}$, which gives the restorations of the separated sources and $\boldsymbol{W}$ is a demixing matrix computed by a BSS procedure. After trying out in a preliminary study the most popular linear BSS procedures (Bell \& Sejnowski, 1995; Belouchrani et al., 1997; Cardoso, 1999; Hyvärinen, 1999; Ziehe \& Muller,1998; Ziehe et al., 2004) for their ability to localize the epileptic foci by our method, Infomax (Bell \& Sejnowski, 1995) was chosen as being the most suitable. The Infomax method was used to compute $W$ for each $60 \mathrm{~s}$ time window and then a topographic map of the restorations $\left\{\hat{s}_{i}(t)\right\}_{i=1}^{n}(1)$ was created by a backprojecting technique (Makeig et al., 1997):

$$
\mathbf{x}_{b p}^{i}(t)=\left(\mathbf{W}_{i}^{(-1)}\right)^{T} \hat{s}_{i}(t)
$$

where $T$ denotes transpose operation, $\boldsymbol{W}_{i}^{(-1)}$ is the $i^{\text {th }}$ column of $\boldsymbol{W}^{-1}$ being the inverse of the demixing matrix $W$ and $\mathbf{x}_{b p}^{i}(t)$ is a back-projected vector comprising activities $\mathbf{x}_{b p j}^{i}(t)$ at locations $\mathrm{j}=1,2, \ldots, \mathrm{n}$ on the scalp implied by the restoration $\hat{s}_{i}(t)$.

Following Jung et al (2001), for each EEG time window and for each $\hat{s}_{i}(t)$, a unique time instance is computed:

$$
\begin{gathered}
t_{i}^{*}=\arg \max _{t} \hat{\operatorname{Va}} a r_{b p}^{i}(t), \\
\hat{\operatorname{Var}} r_{b p}^{i}(t)=\frac{1}{n} \sum_{j 1=1}^{n}\left(x_{b p_{j 1}}^{i}(t)-\frac{1}{n} \sum_{j 2=1}^{n} x_{b p_{j 2}}^{i}(t)\right)^{2},
\end{gathered}
$$

where $\hat{\operatorname{Var}} r_{b p}^{i}(t)$ denotes sample variance of the back projected activities $\mathbf{x}_{b p j}^{i}(t) \quad j=1,2, \ldots, n$. Following this, a vector is constructed:

$$
B_{b p}^{i}=\left[x_{b p_{1}}^{i}\left(t_{i}^{*}\right), \ldots, x_{b p n}^{i}\left(t_{i}^{*}\right)\right]^{T}
$$

comprising the back projected activities at time $t_{i}{ }^{*}$ for each EEG time window and each $\hat{s}_{i}(t)$.

The topographic maps can be conceptually grasped as depicting the spatial manifestation of a given separated source. Inter-ictal epileptic activity in focal epilepsy is thought to be restricted to a small localized brain volume. Therefore, it is assumed that the back projected quantities $\left\{x_{b p j}^{i}\left(t_{i}{ }^{*}\right)\right\}_{j=1}^{n}$ at the locations $j=1,2, \ldots, n$ on the scalp are not likely to represent epileptic activity if 'hot' regions are relatively widely distributed. Hence, $\mathrm{Pe}(i)$ is defined: 


$$
P e(i)=\frac{\max _{j}\left(x_{b p j}^{i}\left(t^{*}\right)\right)}{\sum_{j=1}^{n} x_{b p j}^{i}\left(t^{*}\right)} \times 100
$$

as a measure of the likelihood of $B_{b p}^{i}$ to represent epileptic activity and it is proposed to define and reject $B_{b p}^{i}(5)$ as being non epileptic, if $P e(i)<10 \%$. For illustration, in Figure 2a the restorations $\left\{\hat{s}_{\mathrm{i}}(\mathrm{t})\right\}_{i=1}^{n}$ for the $\left\{x_{j}(t)\right\}_{j=1}^{n}$ shown in Figure 1 are presented. An expert EEG analyst would recognize epileptic-like activity in $\hat{s}_{i}(t)$ restorations: $\mathrm{i}=5-8,13-15$ and 19 , most of which indeed display highly non uniform heat maps of the values of $\left\{x_{b p j}^{i}\left(t_{i}^{*}\right)\right\}_{j=1}^{n}$ (5) (Figure 2b). The latter are reflected by large values of their $P e(i)$ (6) (in the range of 15$61 \%$ ), which indicate a strong orientation to a specific location on the scalp. It is again noteworthy that such orientation in this patient involves diverse scalp locations (temporal, and occipital, bilaterally). It should also be noted that localized heat maps and high $\mathrm{Pe}(i) \mathrm{s}$ can also be seen in restorations such as $i=17$ and 21 , that are seemingly not associated with epileptic activity. The latter may be artifacts or other brain signals that are not expected to reoccur systematically and with a fixed spatial representation throughout the pre-ictal period, and as such will not be grouped by the clustering of the BSS topographic maps, described in the next section.

\subsection{Clustering of the BSS topographic maps}

After computing $B_{b p}^{i} \mathrm{~s}(5)$ for all overlapping $60 \mathrm{~s}$ windows obtained from several preseizure time interval recordings for a single patient, all $B_{b p}^{i} \mathrm{~s}$ having $\mathrm{Pe}(i)>10 \%$ are collated as being potentially epileptic. Similar $B_{b p}^{i} \mathrm{~s}$ are then grouped by a cluster analysis method. In preliminary trials, an attractive accuracy in focus localization was found by using unsupervised fuzzy clustering analysis, employing a correlation distance (Gath \& Geva, 1989):

$$
d\left(B_{b p}^{*}, B_{b p}^{* *}\right)=1-\frac{B_{b p}^{*}{ }^{T} B_{b p}^{* *}}{\left\|B_{b p}^{*}\right\|\left\|B_{b p}^{* *}\right\|}
$$

where $B_{b p}^{*}$ and $B_{b p}^{* *}$ denote arbitrary vectors of $B_{b p}^{i}(5)$ and $\|\bullet\|$ is the L2 norm of the vectors. The efficacy of $d\left(B_{b p}^{*}, B_{b p}^{* *}\right)$ in this case may stem from its invariance to rotation and dilation (Duda et al., 2000), which seems to be critical for the grouping of epileptic $B_{b p}^{i} \mathrm{~s}$.

The cluster analysis procedure results in $\mathrm{K}$ groups of $B_{b p}^{i} \mathrm{~s}$ ( $\mathrm{K}$ being set by a validation criterion explained in Gath \& Geva (1989)), for each of which the following statistics are computed:

1. The averaged sample variance 


$$
\bar{V}^{(k)}=\frac{1}{n} \operatorname{trace}\left(\hat{\Sigma}_{b p}^{(k)}\right), k=1,2, \ldots, K
$$

where $\hat{\Sigma}_{b p}^{(k)}$ is the sample covariance matrix of $B_{b p}^{i}$ 's in group k.

2. The average $P e(i)$ value (6) of $B_{b p}^{i} \mathrm{~s}$ in group $\mathrm{k}$, denoted by $\overline{P e}^{(k)}, k=1, \ldots, K$.

The values of these two statistics for $m$ members of each group are arranged in descending order, after which Score $\bar{V}^{(k)}$ and Score $\overline{P e}^{(k)}$ are defined, assigning score 1 for the largest value and $K$ for the smallest.

The two scores are joined in an index:

$$
I_{e . a}^{(k)}=\frac{K+\operatorname{Score} \overline{P e}^{(k)}-\operatorname{Score}^{(k)}}{2(K-1)+1}
$$

having a range of $0 \leq I_{e . a}^{(k)} \leq 1$, which is normalized for each patient:

$$
\tilde{I}_{e . a}^{(k)}=\frac{I_{e . a}^{(k)}-I_{e . a}^{\min }}{I_{e . a}^{\max }-I_{e . a}^{\min }}
$$

with $I_{e . a}^{\min }=\min _{k}\left\{I_{e . a}^{(k)}\right\}, I_{e . a}^{\max }=\max _{k}\left\{I_{e . a}^{(k)}\right\}$. The normalized index $\tilde{I}_{e . a}^{(k)}$ has similar extrimum values of 0 and 1 for all patients. The expression $I_{e . a}^{(k)}$ (9) reflects the accepted medical knowledge for the pre-ictal activity. For a group (cluster) of $B_{b p}^{i} \mathrm{~s}$ which is most likely to represent epileptic activity (henceforth: 'epileptic group'), $I_{e . a}^{(k)}$ tends to have a small value, implying a low value of $\bar{V}^{(k)}(8)$ and/or a high value of $\overline{\mathrm{Pe}}^{(k)}$.

The means of the $B_{b p}^{i} \mathrm{~s}(5)$ within the groups are then computed, which are denoted by:

$$
\bar{B}_{b p}^{(k)}=\left[\bar{x}_{b p_{1}}^{(k)}, \ldots, \bar{x}_{b p}^{(k}\right]^{T}, k=1,2, \ldots, K
$$

Finally, a number $J^{(k)}$ is calculated:

$$
J^{(k)}=\arg \max _{j}\left\{\bar{x}_{b p j}^{(k)}\right\}_{j=1}^{n},
$$

being the EEG scalp electrode number (1-27) having the highest mean activity value $\bar{x}_{b p j}^{(k)}(5)$ within group $k$. The number $J^{(k)}$ for the 'epileptic group' is likely to indicate the EEG electrode with the closest orientation to the epileptic brain focus and thus, the most appropriate to represent its reflection upon the surface of the scalp. 


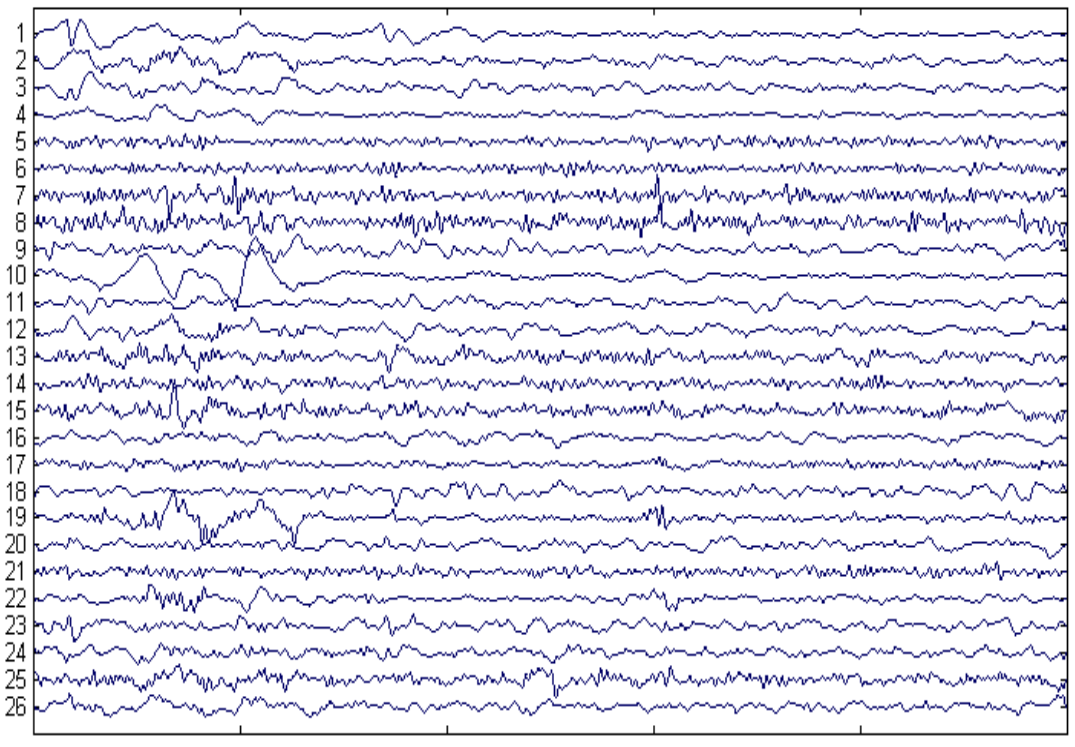
$\begin{array}{lllll}P e(1)=12 & P e(2)=11 & P e(3)=9 & P & (\text { a) } \quad\end{array}$

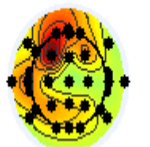

$P e(8)=27$

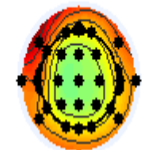

$P e(9)=9$

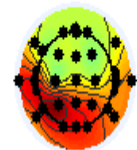

$P e(10)=23$

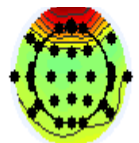

$P e(11)=8$

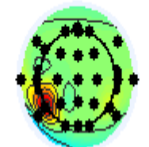

$P e(12)=11$

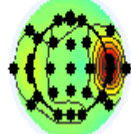

$P e(13)=16$

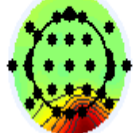

$P e(14)=42$
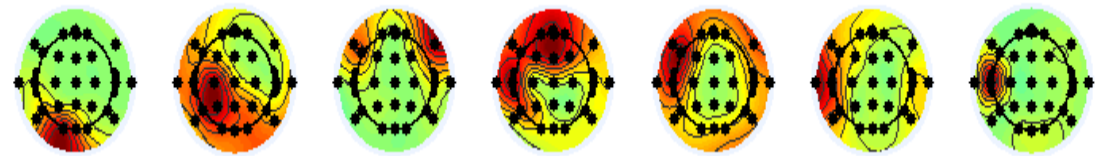

$P e(15)=15$

$P e(16)=13$

$P e(17)=32$

$P e(18)=13$

$P e(19)=27$

$P e(20)=13$

$P e(21)=46$
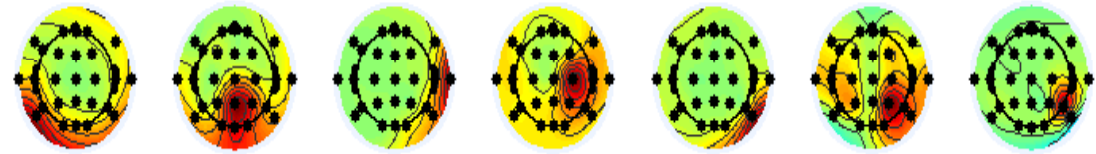

$P e(22)=26$

$\mathrm{Pe}(23)=19$

$P e(24)=17$

$P e(25)=13$

$P e(26)=21$
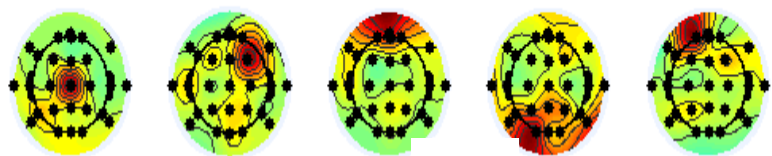

(b)

Fig. 2. BSS results for patient \# 2. (a) BSS restorations $\hat{\mathrm{s}}_{\mathrm{i}}(t), i=1, \ldots, n$ obtained from $\left\{x_{j}(t)\right\}_{j=1}^{n}$ in Figure 1. (b) Heat maps of the values $x_{b p j}^{i}\left(t_{i}{ }^{*}\right)(5)$ at points $j=1,2, \ldots, n$ on the scalp. $\mathrm{Pe}(i)$ values (6) are shown above each map. 
The model's results for patient \#2 are shown in Figure 3. Figure 3a presents the heat maps of $\bar{B}_{b p}^{(k)}(11)$ for the three of seven groups identified by the cluster analysis, having the smallest values of the index $I_{e . a}^{(k)}(10)$, given in parentheses over the maps. They are reminiscent of restorations 5,6 and 7 of Figure 2, the latter being members in clusters $K=1,2$ and 3, respectively. For completeness, Figures $3 b, c$ present the values of $\bar{V}^{(k)}$ and $\overline{P e}^{(k)}$, respectively, for all seven groups. The first group $(K=1)$, with $I_{e . a}^{(k)}=0$, is the "epileptic group". The number $J^{(1)}$ computed by (12) suggests that the epileptic focus is mostly reflected in electrode T5. Groups 2 and 3 are projections to the contra-lateral hemisphere, which interestingly scored higher than the map centered over T3, yet trailed the 'epileptic group' considerably. Non-epileptic restorations in this patient (e.g. numbers 17 and 21 in Figure 2) either did not form clusters or formed clusters with high $I_{e . a}^{(k)}$ values.

$1(0)$
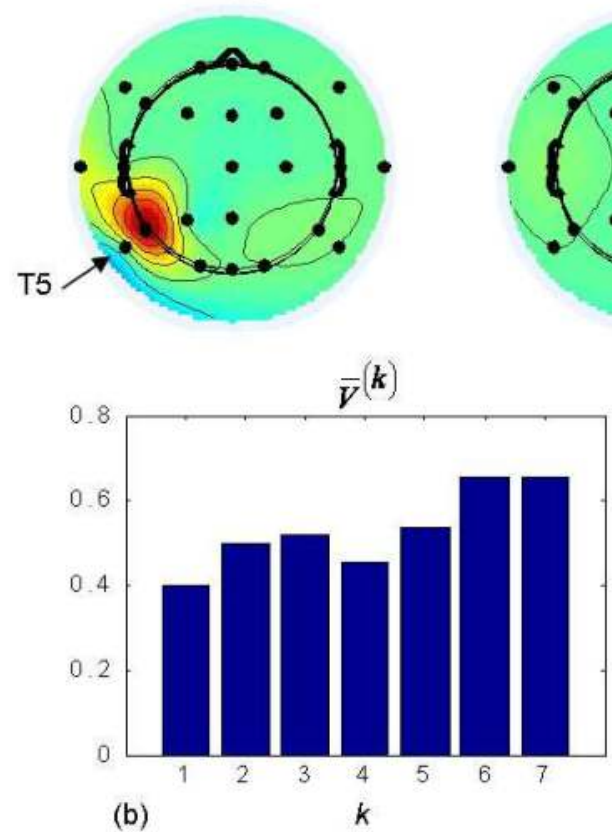

$2(0.25)$

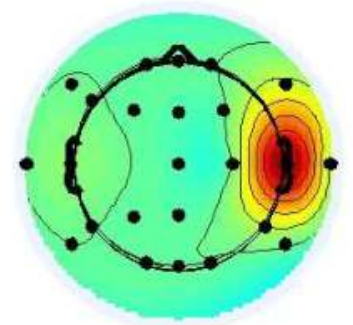

(a)
$3(0.42)$

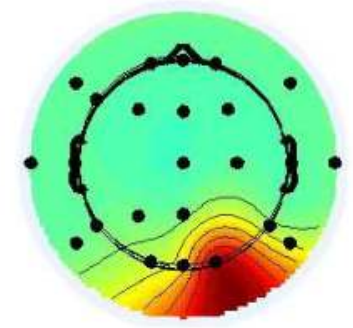

$\overline{P e}(k)$

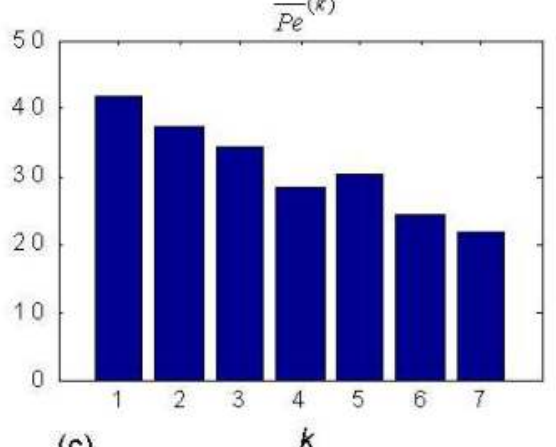

(c)

Fig. 3. Localization of the epileptic focus for patient \#2. (a) Heat maps of $\bar{B}_{b p}^{(k)}$ for the three groups having the smallest values of $\tilde{I}_{e . a}^{(k)}(10)$ (shown in parentheses above maps); (b) and (c) The averaged sample variance $\bar{V}^{(k)}(8)$ and the average $P e(i)$ value (6) of $B_{b p}^{i}$ s in group k, $\overline{P e}{ }^{(k)}$, respectively, for all seven groups identified for this patient. Map 1 is that of the 'epileptic group' and location T5 (arrow) came out as closest to the source.

\section{Experimental results}

The results obtained by the proposed method on 12 patients are now presented. Table 1 lists the relevant clinical information. 


\begin{tabular}{|c|c|c|c|c|c|c|c|}
\hline $\begin{array}{c}\text { Patient } \\
\text { № }\end{array}$ & $\begin{array}{c}\text { Age } \\
(\mathrm{y})\end{array}$ & Gender & $\begin{array}{c}\text { Age at } \\
\text { disease } \\
\text { onset (y) }\end{array}$ & $\begin{array}{c}\text { Seizure } \\
\text { frequency* }\end{array}$ & $\begin{array}{c}\text { Validation method } \\
\text { for epileptic focus } \\
\text { localization }\end{array}$ & $\begin{array}{c}\text { Number } \\
\text { of seizures } \\
\text { analyzed }\end{array}$ & $\begin{array}{c}\text { Range (mean) } \\
\text { of pre-ictal } \\
\text { period } \\
\text { (minutes) }\end{array}$ \\
\hline 1 & 17 & $\mathrm{~F}$ & 9 & $6 / \mathrm{m}$ & IR & 3 & $4.5-6.4(5.3)$ \\
\hline 2 & 8 & $\mathrm{M}$ & 3 & $2 / \mathrm{w}$ & IR & 5 & $7.4-35.3(17.5)$ \\
\hline 3 & 8.5 & $\mathrm{M}$ & 7 & $15 / \mathrm{d}$ & MRI & 2 & $3.8-9.8(6.8)$ \\
\hline 4 & 12 & $\mathrm{~F}$ & 1 & $10 / \mathrm{d}$ & IR & 2 & $10-10.2(10.1)$ \\
\hline 5 & 16 & $\mathrm{~F}$ & 6 & $1 / \mathrm{d}$ & MRI & 5 & $0.8-5(2.3)$ \\
\hline 6 & 20 & $\mathrm{M}$ & 0.7 & $2 / \mathrm{d}$ & MRI, IR & 2 & $2.2-9.3(5.7)$ \\
\hline 7 & 26 & $\mathrm{~F}$ & 19 & $2 / \mathrm{d}$ & MRI & 7 & $5.7-12.9(7.8)$ \\
\hline 8 & 19 & $\mathrm{~F}$ & 6 & $2 / \mathrm{d}$ & SPECT & 2 & $6.7-9.6(8.2)$ \\
\hline 9 & 54 & $\mathrm{~F}$ & 0.5 & $3 / \mathrm{w}$ & MRI & 5 & $5.5-26.6(10.7)$ \\
\hline 10 & 22 & $\mathrm{~F}$ & 2 & $6 / \mathrm{w}$ & MRI, IR & 3 & $5.1-13.8(9.8)$ \\
\hline 11 & 34 & $\mathrm{M}$ & 4 & $2 / \mathrm{d}$ & MRI, IR & 7 & $2-5.5(3.6)$ \\
\hline 12 & 54 & $\mathrm{M}$ & 50 & $2 / \mathrm{w}$ & MRI & 2 & $9.4-11.5(10.5)$ \\
\hline
\end{tabular}

Table 1. Patients' clinical details. * At the time of video monitoring (per day $(\mathrm{d})$, week $(\mathrm{w})$, month (m)). IR - Invasive Recordings.

The clinical material contains five cases (patients 1-4, 7) with focal lateral temporal lobe epilepsy, two cases (patients 8,9) with mesial temporal lobe epilepsy, three (patients 10-12) with frontal lobe epilepsy and two (patients 5,6) with a focus in the parietal lobe. The location of the suspected epileptic brain region was determined by the clinical neurophysiologist and correlated with brain MRI images or inter-ictal SPECT. For six cases (\# 1, 2, 4, 6, 10, 11) additional validation for the localization of the epileptic region was obtained using invasive recordings (IR) by intracranial (mostly sub-dural) electrodes implanted prior to surgical resection of the epileptic brain region. Two to seven scalp EEG strips were obtained from each patient (Table 1), all ending at the seizure's onset. The clinical onset time of seizures was determined by the clinical neurophysiologist based on video-EEG recorded data (seizure onset was determined as the first sign of a seizure - either clinically or neurophysiologicaly). In figure 4, the IR verification method results are shown for patient \#2.

Figure 5a illustrates an MRI scan of patient \#5 showing a suspected dysplastic cortical lesion in the left parietal region (arrow). Cortical dysplasia is a developmental cortical lesion in which neurons fail to migrate properly and is frequently associated with epilepsy. The epileptic region in many cases is in close proximity to the dysplastic brain region. In Figure $5 b$, the results for focal localization (heat map of $\bar{B}_{b p}^{(k)}(11)$ corresponding to the smallest $\tilde{I}_{e . a}^{(k)}$ (10)) are shown, pointing on the same left parietal origin.

In Table 2 the result obtained for the 12 study patients are summarized. In all patients, the epileptic source was lateralized correctly. This achievement is not trivial when being reminded that it is not based on any temporal relations but only on average spatial spread. In 9 cases (patients №s 1-5, 7,9,10 and 12) the smallest value of $\tilde{I}_{e . a}^{(k)}(10)$ for the heat map of $\bar{B}_{b p}^{(k)}$ was obtained with correct identification of $J^{(1)}$ as being closest to the focus. 
Automated Non-Invasive Identification and Localization of

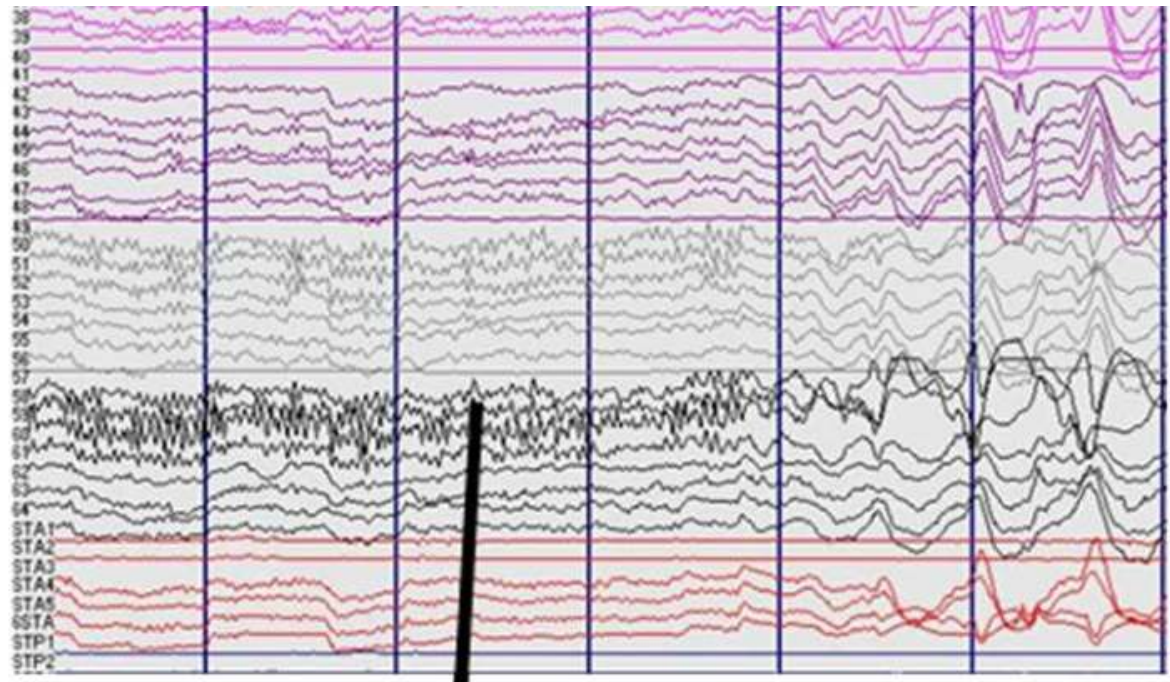

a

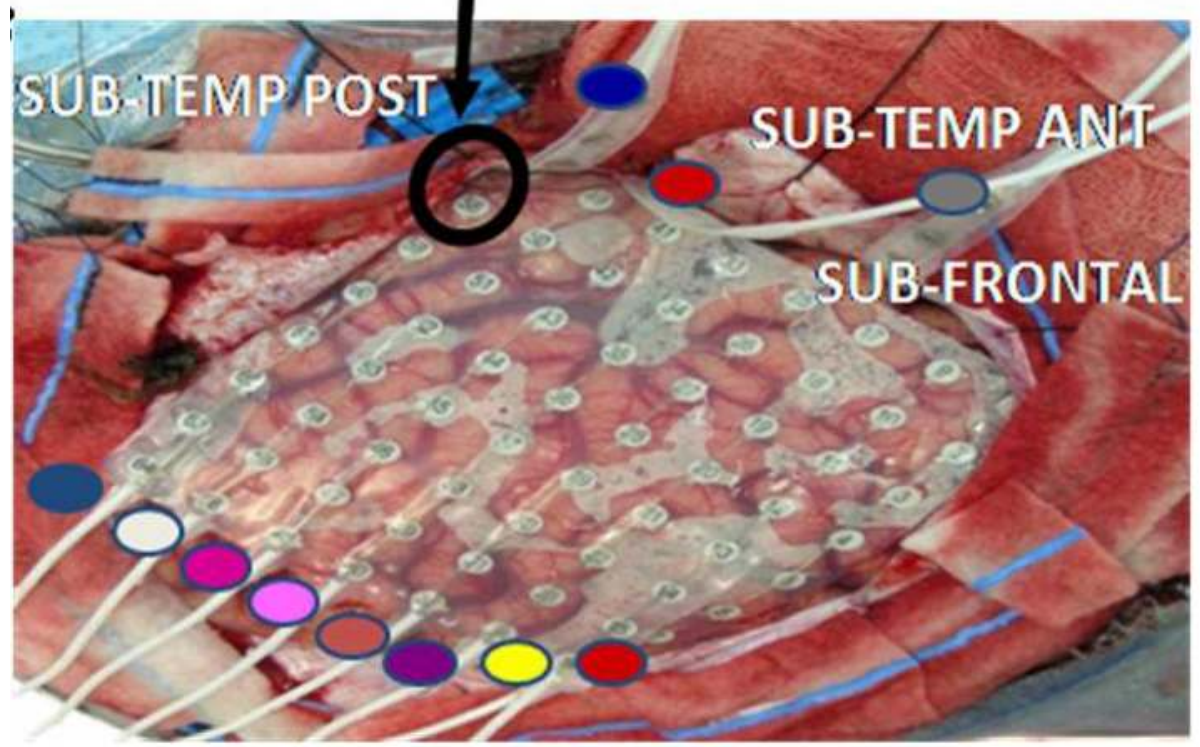

b

Fig. 4. Verification of focus localization for patient \#2. Invasive recordings (a) from a grid of sub-dural electrodes implanted over the left anterior lateral temporal lobe of the patient (b). This focus is closest to the T5 scalp electrode, the same one identified by the proposed method (see Fig. 3a). 

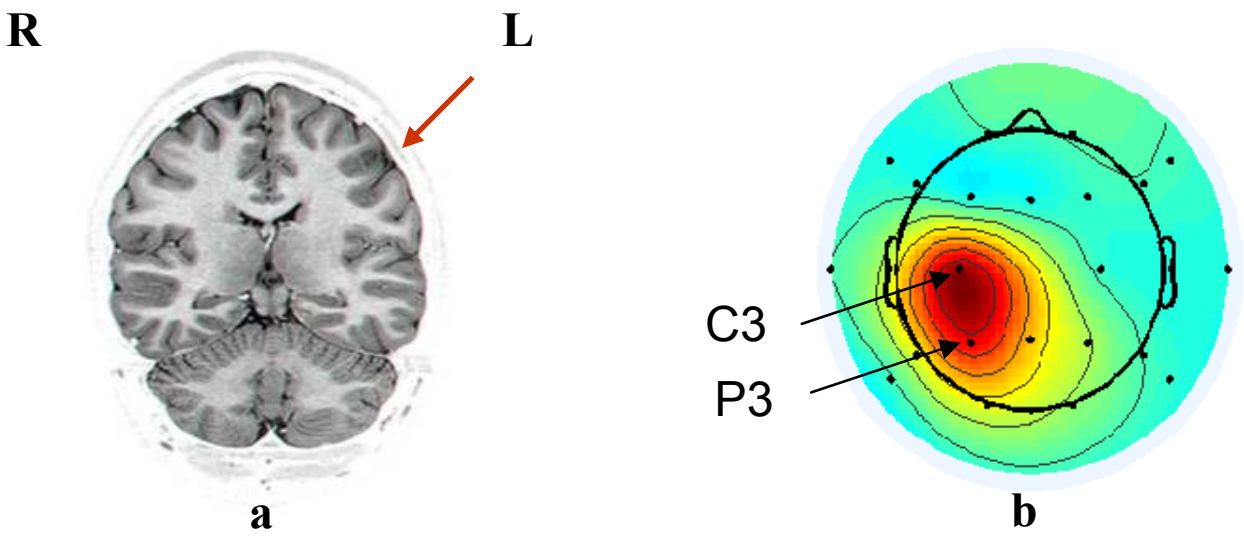

Fig. 5. Focus localization for patient \#5. (a) MRI brain scan. The arrow points on the left parietal region, under surface electrodes P3,C3. (b) Heat map of $\bar{B}_{b p}^{(k)}$ (11) of the group with the lowest value of $\tilde{I}_{e . a}^{(k)}(10)$, pointing to the same left parietal origin and two surface electrodes.

For the other 3 patients (marked by *) questionable consistency was obtained with clinical observation. In patient 6 , the center of the detected epileptic cluster was not focused around one specific electrode but was equally distant from three surface electrodes and hence the $\tilde{I}_{e . a}^{(k)}$ of the actual closest scalp electrode was not the lowest (although having a relatively small value). Patient 8 had mesial temporal lobe epilepsy (MTLE) which was difficult to localize clinically. In patients with this infliction, interictal epileptiform discharges arising from restricted and deep cortical areas such as the hippocampus may be hardly distinguishable from background EEG activity or may even remain undetected on scalp EEG recordings (i.e. Nayak et al., 2004). Hence, the results of the method concerning this patient were defined as questionable. More cases with MTLE need be analyzed in order to assess the method's applicability to this major variant of focal epilepsy. Patient \#11 had a frontal focus (closest to FP1) and hence it was harder to detect due to blinking artifacts affecting that region. This problem has recently been successfully resolved using a constrained BSS procedure (Shoker et al., 2005). 
Automated Non-Invasive Identification and Localization of

Focal Epileptic Activity by Exploiting Information Derived from Surface EEG Recordings

\begin{tabular}{|c|c|c|c|}
\hline \multicolumn{2}{|c|}{ Present findings } & \multirow[b]{2}{*}{ clinical localization } & \multirow[b]{2}{*}{ Patient № } \\
\hline $\begin{array}{l}\text { Epileptic heat maps } \\
\text { having smallest } \tilde{I}_{e . a}^{(k)}\end{array}$ & $J^{(1)}$ & & \\
\hline & $\mathrm{T} 3$ & Left Temporal (T3-T5) & 1 \\
\hline & T5 & Left temporal (T5) & 2 \\
\hline & $\mathrm{T} 4$ & Right temporal (T4-T6) & 3 \\
\hline & A1 & Left temporal (A1-T3) & 4 \\
\hline & $\mathrm{C} 3$ & Left centro- parietal (C3-P3) & 5 \\
\hline & $\mathrm{F} 4$ & Right centro- parietal (C4-P4) & $6^{*}$ \\
\hline & T3 & Left temporal lobe (T3) & 7 \\
\hline & T3 & Left mesial temporal lobe & $8^{*}$ \\
\hline & T3 & Left temporal (T3) & 9 \\
\hline & F7 & Left fronto- parietal (F7) & 10 \\
\hline & T3 & Left fronto-temporal (F9) & $11^{*}$ \\
\hline & F8 & Right frontal (F4-F8) & 12 \\
\hline
\end{tabular}

Table 2. Patients' focus localization 


\section{Summary and conclusions}

In this chapter a method is introduced for localizing an epileptic source, exploiting information from scalp electroencephalographic (EEG) recordings. Blind source separation (BSS) and cluster analysis are combined with generally known pathological features of the epileptic focus. The combination of these unsupervised methods allows us to detect hidden epileptic patterns, at times, unrevealed by other means. The proposed method may help minimize the number and extent of invasive electrodes used in ambiguous cases, when surface EEG recordings do not offer a definite diagnosis. It should be stressed that the method is not intended to replace the expert clinical evaluation; rather, it has been designed to focus the clinician's attention on regions which are more likely to harbor the source of the epileptic activity and which may merit a more careful anatomical (using MRI) or neurophysiological evaluation.

In the derivation of the method, we first describe the data acquisition and preprocessing of the EEG signals, steps that have crucial influence on the following steps. BSS is then employed for restoration of independent neural and extra-neural components in the EEG signals. The seizure in focal epilepsy is thought to arise in a small brain volume. Based on this assumption, a measure $\mathrm{Pe}(i)$ (6) is derived for the likelihood of the BSS back-projected quantities $B_{b p}^{i}(5)$ to represent epileptic activity. Then, similar $B_{b p}^{i} \mathrm{~s}$ with above-threshold $P e(i)$ are grouped by a cluster analysis method. Finally, an index $\tilde{I}_{e . a}^{(k)}(10)$ is derived, which indicates the group of $B_{b p}^{i}$ s most likely to represent epileptic activity. By identifying this group and its dominant electrode we actually localize the epileptic focus.

We then present results of the method, as applied on twelve patients having prior invasive neuro-physiological verification of the actual localization of the epileptic focus. Correct lateralization was achieved for all 12 patients. For nine of the twelve patients the method pinpointed the scalp location closest to epileptic focus. For the other three patients, questionable consistency with clinical observation was obtained. Being automated and unsupervised, the method is a promising tool for pre-surgical evaluation.

\section{References}

Baumgartner, C. Lindinger, G. Ebner, A. Aull, S. Serles, W. Olbrich, A. Lurger, S. Czech, T. Burgess, R. \& Lüders, H. (1995). Propagation of interictal epileptic activity in temporal lobe epilepsy. Neurology, Vol.45, pp. 118-22

Bell, AJ. \& Sejnowski, TJ. (1995). An information-maximization approach to blind separation and blind deconvolution. Neural Computation, Vol.7, pp. 1129-1159

Belouchrani, A. Abed-Meraim, K. Cardoso, JF. \& Moulines, E. (1997). A blind source separation technique. IEEE Transactions on Signal Processing, Vol.45, pp. 434-444

Bezdek, JC. (1981). Pattern recognition with fuzzy objective function algorithms, Plenum, New York, USA

Blume, WT. Holloway, GM. \& Wiebe, S. (2001). Temporal epileptogenesis: localizing value of scalp and subdural interictal EEG data. Epilepsia, Vol.42, pp. 508-514

Bouzida, N. Peyrodie, L. \& Vasseur, C. (2005). ICA and a gauge of filter for the automatic filtering of an EEG signal Proceedings of theIEEE International Joint Conference on Neural Networks, pp. 2508-2513, Montreal, Canada, July 31-August 4, 2005 
Cardoso, JF. (1999). High-order contrasts for independent component analysis. Neural computation, Vol.11, pp. 157-192

Chang, KM. \& Po, PC. (2005). Meditation EEG interpretation based on novel fuzzy-merging strategies and wavelet features. Biomedical Engineering Applications, Vol.17, pp. 167175

Chiappa, S. Barber D (2005) Generative independent component analysis for EEG classification. Proceedings of the European Symposium on Artificial Neural Networks, ESANN' 2005, Burges, pp. 297-302.

Cichocki, A. Shishkina SL, Mushac T, Leonowicz Z (2005) EEG filtering based on blind source separation (BSS) for early detection of Alzheimer's disease. Clinical Neurophysiology 116:729-737.

Corsini, J. Shoker L, Sanei S, Alarcón G (2006) Epileptic seizure predictability from scalp EEG incorporating constrained blind source separation. IEEE Transactions on Biomedical Engineering 53:790-799.

Delorme, A. Makeig S (2004) EEGLAB: an open source toolbox for analysis of single-trial EEG dynamics including independent component analysis. Journal of Neuroscience Methods 134:9-21.

Duda, RO. Hart PE, Stork DG (2000) Pattern Classification, 2d Ed. Wiley, New York.

Ebersole, JS. (2000) Noninvasive localization of epileptogenic foci by EEG source modeling. Epilepsia 41:24-33.

Faul, S. Marnane L, Lightbody G, Boylan G, Connolly S (2005) A method for the blind separation of sources for use as the first stage of a neonatal seizure detection system. Proceedings of IEEE International Conference on Acoustics, Speech, and Signal Processing ICASSP '05, Philadelphia pp. 409-412.

Frank, RM. Frishko GA (2007) Automated protocol for evaluation of electromagnetic component separation (APECS): application of a framework for evaluating statistical methods of blink extraction from multichannel EEG. Clinical Neurophysiology 118:80-97.

Gath, I. \& Geva, AB. (1989). Unsupervised optimal fuzzy clustering. IEEE Transactions on Pattern Analysis and Machine Intelligence, Vol.7, pp. 773-781

Gavaret, M. Trébuchon, A. Bartolomei, F. Marquis, P. McGonigal, A. Wendling, F. Regis, J. Badier, JM. Chauvel, P. (2009). Source localization of scalp-EEG interictal spikes in posterior cortex epilepsies investigated by HR-EEG and SEEG. Epilepsia, Vol.50, pp. 276-289

Geva, AB. Kerem, DH. (1998). Forecasting generalized epileptic seizures from the EEG signal by wavelet analysis and dynamic unsupervised fuzzy clustering. IEEE Transactions on Biomedical Engineering, Vol.45, pp. 1205-1216

Hallez, H. Vanrumste, B. Grech, R. Muscat, J. De Clercq, W. Vergult. A. D'Asseler, Y. Camilleri, KP. Fabri, SG.Van Huffel, S. \& Lemahieu, I. (2007). Review on solving the forward problem in EEG source analysis. Journal of Neuroengineering and Rehabilitation, Vol.4, p. 46 (29 pp.)

Hesse, CW. \& James, CJ. (2005). Tracking epileptiform activity in the multichannel ictal EEG using spatially constrained independent component analysis, Proceedings of the 27th Annual International Conference of the IEEE Engineering in Medicine and Biology Society, pp. 2067-2070, Shanghai, China, September 1-3, 2005 
Hyvärinen, A. (1999). Fast and robust fixed-point algorithms for independent component analysis. IEEE Transactions on Neural Networks, Vol.10, pp. 626-634

Hyvärinen, A. \& Oja, E. (2000). Independent component analysis: Algorithms and applications. Neural Networks, Vol.13, pp. 411-430

Hyvärinen, A. Karhunen, J. Oja, E. (2001). Independent component analysis, Wiley, New York, USA

James, CJ. \& Lowe, D. (2000). Using independent component analysis \& dynamical embedding to isolate seizure activity in the EEG. Proceedings of the $22^{\text {nd }}$ annual international conference of the IEEE Engineering in Medicine and Biology Society, pp. 1329-1332, Chicago, Illinois, USA, July 23-28, 2000

James, CJ. \& Gibson, OJ. (2003). Temporally constrained ICA: an application to artifact rejection in electromagnetic brain signal analysis. IEEE Transactions on Biomedical Engineering, Vol.50, pp. 1108-1116

Jarchi, D. Boostani, R. Taheri, M. \& Sanei, S. (2009). Seizure source localization using hybrid second order blind identification and extended rival penalized competitive learning algorithm. Biomedical Signal Processing and Control, Vol.4, pp. 108-117

Jing, M. \& Sanei, S. (2007). A novel constrained topographic independent component analysis for separation of epileptic seizure signals. Computational Intelligence and Neuroscience, Vol.1, pp. 1-7

Jung, TP. Makeig, S. Mckeown, MJ. Bell, AJ. Lee, TW. \& Sejnowski, TJ. (2001). Imaging brain dynamics using independent component analysis. Proceedings of the IEEE, Vol.897, pp. 1107-1122

Kobayashi, K. Nakahori, CJ. Akiyama, T. \& Gotman, J. (1999). Isolation of epileptiform discharges from unaveraged EEG by independent component analysis. Clinical neurophysiology, Vol.110, pp. 1755-1763

Lantz, G. Spinelli, L. Seeck, M. Grave de Peralta, R. Sottas, C. \& Michel, C. (2003). Propagation of interictal epileptiform activity can lead to erroneous source localizations: a 128-channel EEG mapping study. Journal of Clinical Neurophysiology Vol.20, pp. 311-319

Latif, MA. \& Sanei, S. (2005). Localization of brain abnormal signal sources using blind source separation, Proceedings of the 27th Annual International Conference of the IEEE Engineering in Medicine and Biology Society, pp. 1170-1172, Shanghai, China, September 1-3, 2005

Liu, H. Hild, KE. Gao, JB. Erdogmus, D. Príncipe, JC. Chris, J. (2004). Evaluation of a BSS algorithm for artifacts rejection in epileptic seizure detection, Proceedings of the 26th Annual International Conference of the IEEE Engineering in Medicine and Biology Society, pp. 91-94, San Francisco, California, USA, September 1-5, 2004

Makeig, S. Jung, TP. Bell, AJ. Mckeown, MJ. Ghahremani, D. \& Sejnowski, TJ. (1997). Blind separation of auditory event-related brain responses into independent components. Proceedings of the National Academy of Sciences, Vol.94, pp. 10979-10984

Makeig, S. Westerfield, M. Jung, TP. Covington, J. Townsend, J. Sejnowski, TJ. \& Courchesne, E. (1999). Functionally independent components of the late positive event-related potential during visual spatial attention. The Journal of Neuroscience, Vol.19, pp. 2665-2680 
Makeig, S. Delorme, A. Westerfield, M. Jung, TP., Townsend, J. Courchesne, E. \& Sejnowski, TJ. (2004). Electroencephalographic brain dynamics following manually responded visual targets. Public Library of Science Biology, Vol. 2, pp. 747-762

Meier, R. Dittrich, H. Schulze-Bonhage, A. Aertsen, A. (2008). Detecting epileptic seizures in long-term human EEG: a new approach to automatic online and real-time detection and classification of polymorphic seizure patterns. Journal of Clinical Neurophysiology, Vol.25, pp. 1-13

Merlet, I. \& Gotman, J. (2001). Dipole modeling of scalp electroencephalogram epileptic discharges: correlation with intracerebral fields. Clinical Neurophysiology, Vol.112, pp. $414-430$

Michel, C. Lantz, G. Spinelli, L. Grave de Peralta, R. Landis, T. \& Seeck, M. (2004). 128channel EEG source imaging in epilepsy: clinical yield and localization precision. Journal of Clinical Neurophysiology, Vol.21, pp. 71-83

Nunez, PL. \& Srinivasan, R. (2006). Electric fields of the brain: The Neurophysics of EEG, Oxford University Press, New York, USA

Nayak, D. Valentin, A. Alarcon, G. Garcia Seoane, JJ. Brunnhuber, F. Juler, J. Polkey, CE. \& Binnie, CD. (2004). Characteristics of scalp electrical fields associated with deep medial temporal epileptiform discharges. Clinical Neurophysiology, Vol.115, pp. 1423-35

Pascual-Marqui. RD. (2002). Standardized low resolution brain electromagnetic tomography (sLORETA): technical details. Methods and Findings in Experimental $\mathcal{E}$ Clinical Pharmacology, Vol.24D, pp. 5-12

Proakis, JG. \& Manolakis, DG. (2007). Digital signal processing, Pearson Prentice-Hall, New Jersey, USA

Richards, JE. (2004). Recovering dipole sources from scalp-recorded event-related-potentials using component analysis: principal component analysis and independent component analysis. International Journal of Psychophysiology, Vol.54, pp. 201-220

Sanei, S. \& Chambers, JA. (2007). EEG Signal Processing, Wiley, New York, USA

Sepulveda, F. Meckes, M. \& Conway, BA. (2004). Cluster separation index suggests usefulness of non-motor EEG channels in detecting wrist movement direction intention. Proceedings of the IEEE conference on cybernetics and intelligent systems, pp. 943-947, Singapore, December 1-3, 2004

Shoker. L, Sanei, S. Wang, W. \& Chambers, JA. (2005). Removal of eye blinking artifact from the electro-encephalogram, incorporating a new constrained blind source separation algorithm. Medical \& Biological Engineering \& Computing, Vol. 43 pp. 290 295

Sutherlanda, MT. \& Tanga AC. (2006). Reliable detection of bilateral activation in human primary somatosensory cortex by unilateral median nerve stimulation. Neuroimage, Vol.33, pp. 1042-1054

Vulliemoz, S. Thornton, R. Rodionov, R. Carmichael, DW. Guye, M. Lhatoo, S. McEvoy, AW., Spinelli, L. Michel, CM. Duncan, JS. \& Lemieux, L. (2009). The spatiotemporal mapping of epileptic networks: Combination of EEG-fMRI and EEG source imaging . Neuroimage, Vol.46, pp. 834-843

Xue, Z. Li, J. Li, S. \& Wan, B. (2006) Using ICA to remove eye blink and power line artifacts in EEG. Proceedings 1st international conference on innovative computing, information and control, ICICIC'06, Beijing pp. 107-110 
Zhukov, L. Weinstein, D. \& Johnson, C. (2000). Independent component analysis for EEG source localization. IEEE Engineering in Medicine and Biology, Vol.19, pp. 87-96

Ziehe, A. \& Muller, KR. (1998). TDSEP - an efficient algorithm for blind separation using time structure. Proceedings of conf. on artificial neural networks, ICANN'98, Skovde, pp. $675-680$

Ziehe, A. Lasko, P. Nolte, G. \& Muller, KR. (2004). A fast algorithm for joint diagonalization with non-orthogonal transformations and its application to blind source separation. Journal of Machine Learning Research, Vol.5, pp. 777-800

Zumsteg, D. Friedman, A. Wennberg, RA. \& Wieser, HG. (2005). Source localization of mesial temporal interictal epileptiform discharges: correlation with intracranial foramen ovale electrode recordings. Clinical Neurophysiology, Vol.116, pp. 2810-2818

Zumsteg, D. Friedman, A. Wieser, HG. \& Wennberg, RA. (2006). Source localization of interictal epileptiform discharges: comparison of three different techniques to improve signal to noise ratio. Clinical neurophysiology, Vol. 117, pp. 562-571 


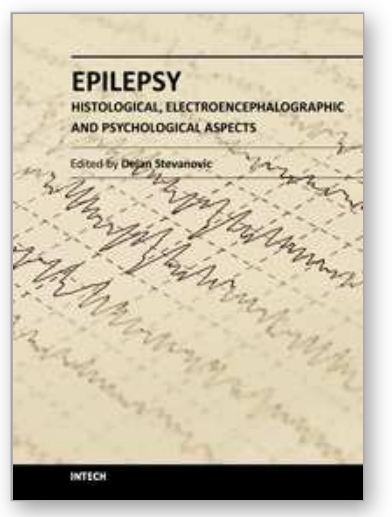

\author{
Epilepsy - Histological, Electroencephalographic and \\ Psychological Aspects \\ Edited by Dr. Dejan Stevanovic
}

ISBN 978-953-51-0082-9

Hard cover, 276 pages

Publisher InTech

Published online 29, February, 2012

Published in print edition February, 2012

With the vision of including authors from different parts of the world, different educational backgrounds, and offering open-access to their published work, InTech proudly presents the latest edited book in epilepsy research, Epilepsy: Histological, electroencephalographic, and psychological aspects. Here are twelve interesting and inspiring chapters dealing with basic molecular and cellular mechanisms underlying epileptic seizures, electroencephalographic findings, and neuropsychological, psychological, and psychiatric aspects of epileptic seizures, but non-epileptic as well.

\title{
How to reference
}

In order to correctly reference this scholarly work, feel free to copy and paste the following:

Amir Geva, Merav Ben-Asher, Dan Kerem, Mayer Aladjem and Alon Friedman (2012). Automated NonInvasive Identification and Localization of Focal Epileptic Activity by Exploiting Information Derived from Surface EEG Recordings, Epilepsy - Histological, Electroencephalographic and Psychological Aspects, Dr. Dejan Stevanovic (Ed.), ISBN: 978-953-51-0082-9, InTech, Available from:

http://www.intechopen.com/books/epilepsy-histological-electroencephalographic-and-psychologicalaspects/automated-non-invasive-identification-and-localization-of-focal-epileptic-activity-by-exploiting-inf

\section{INTECH}

open science | open minds

\section{InTech Europe}

University Campus STeP Ri

Slavka Krautzeka 83/A

51000 Rijeka, Croatia

Phone: +385 (51) 770447

Fax: +385 (51) 686166

www.intechopen.com

\section{InTech China}

Unit 405, Office Block, Hotel Equatorial Shanghai

No.65, Yan An Road (West), Shanghai, 200040, China 中国上海市延安西路65号上海国际贵都大饭店办公楼 405 单元

Phone: +86-21-62489820

Fax: +86-21-62489821 
(C) 2012 The Author(s). Licensee IntechOpen. This is an open access article distributed under the terms of the Creative Commons Attribution 3.0 License, which permits unrestricted use, distribution, and reproduction in any medium, provided the original work is properly cited. 